\title{
PREVALENCIA DE INTERVENCIONES DE TERAPIA OCUPACIONAL EN UN HOSPITAL DE AGUDOS
}

\author{
PREVALENCE OF OCCUPATIONAL THERAPY INTERVENTIONS IN AN ACUTE HOSPITAL
}

\section{Cecilia Caillet-Bois D. ${ }^{1}$, Ma. Eugenia Hernández D²., Pilar Muñoz A. ${ }^{3}$, Sofía Murray P. ${ }^{4}$, Valeria Illán Z. ${ }^{5}$}

\section{Resumen:}

El objetivo del presente trabajo consiste en conocer el proceso de tratamiento de Terapia Ocupacional en las áreas de consultorios externos de Reumatología e internación de Clínica Médica y Unidad de Terapia Intermedia del Hospital Tornú.

Metodología: estudio descriptivo y comparativo, observacional, retrospectivo, transversal, cuantitativo; instrumento: fichas de registro de evaluación y evolución internas de la Residencia.

Resultados: la intervención más prevalente fue: Uso terapéutico de las actividades y ocupaciones con un $41,04 \%$ en Internación y $47 \%$ en Reumatología. Entre estos se destacan las métodos preparatorios con un $56,33 \%$ en Internación y un 84,7 \% en Reumatología. Se observó diferencia significativa en la Interconsulta con $28.32 \%$ en Internación y $5 \%$ en Reumatología.

Por último, el motivo de alta más frecuente en Internación fue alta médica con el $56 \%$ ( $n: 43$ ), seguido de cumplimiento de objetivos con el 16, 4\% (n: 10), y el menos frecuente fue Negativa con el 1,6\% ( $n: 1)$. En Reumatología solo se registraron dos motivos abandono de tratamiento con el 65\% (n: 43) y cumplimiento de objetivos con el $34 \%$ ( $n$ : 22).

Conclusiones: la intervención que más prevaleció en ambas áreas se debe al gran porcentaje de métodos preparatorios, siendo mayor en Reumatología dado que éstos se vinculan con el alivio sintomático.

La Interconsulta no se realizó con la frecuencia necesaria que requiere la complejidad del paciente agudo, por lo que consideramos relevante plantear estrategias de comunicación con el equipo.

\section{Palabras clave}

Terapia Ocupacional, Intervenciones, Hospital de Agudos, proceso de tratamiento.

\footnotetext{
${ }^{1}$ Lic. Terapia Ocupacional, Residente de 2 o año del Htal. Dr. E. Tornú de GCBA

${ }^{2}$ Lic. Terapia Ocupacional, Residente de 3 o año del Htal. Dr. E. Tornú de GCBA

${ }^{3}$ Lic. Terapia Ocupacional, Residente de 30 año del Htal. Dr. E. Tornú de GCBA

${ }^{4}$ Lic. Terapia Ocupacional, Residente de 1 o año del Htal. Dr. E. Tornú de GCBA

${ }^{5}$ Lic. Terapia Ocupacional, Jefa de Residentes del Htal. Dr. E. Tornú de GCBA

Tel. de la Residencia: 005411-4521-1236 int: 193 / terapiaocupacionaltornu@gmail.com
} 


\section{Abstract}

The aim of this study is to know the process of Occupational Therapy treatment given to Rheumatology outpatient, and Clinical and Medical Intensive Care Unit inpatient of Tornú Hospital

Methodology: descriptive, comparative, observational, retrospective, cross-sectional and quantitative instrument; Residence registration evaluation forms and internal evolution.

Results: the most prevalent intervention was the Therapeutic use of Occupations and Activities with $41.04 \%$ in inpatient and $47 \%$ in outpatients (Rheumatology). Among these the Preparatory Methods are highlighted in both areas with a $56.33 \%$ in inpatients and $84.7 \%$ in outpatients (Rheumatology). A significant difference was found in Interconsultation with a $28.32 \%$ in inpatients and $5 \%$ in outpatients (Rheumatology).

Lastly, the most frequent reason for discharge from Occupational Therapy treatment in inpatients was clinical discharge with $56 \%(n=43)$, followed by goal achievement $4 \%(n=10)$, and the least frequent was denial to receive treatment $1,6 \%(n=1)$. Only two reasons were identified in Rheumatology: non-compliance with treatment $65 \%(n=43)$ and goal achievement with $34 \%(n=22)$.

Conclusions: The most prevalent intervention in both areas is due to the large percentage of preparatory methods used, being higher in Rheumatology since these are associated with symptomatic relief.

The Interconsultation was not performed with the frequency required by the acute patient's complexity. According to these results we consider relevant to develop new communication strategies with the team.

\section{Key words}

Occupational Therapy, Interventions, Acute Hospital, Treatment process. 


\section{INTRODUCCIÓN}

La Residencia de Terapia Ocupacional del Hospital Tornú perteneciente al Gobierno de la Ciudad de Buenos Aires, comienza a funcionar a partir de 1998 y actualmente se incluye en los servicios de Clínica Médica, Unidad de Terapia Intermedia, Reumatología, Ortopedia y Traumatología y la Unidad de Cuidados Paliativos. En un trabajo realizado durante el período Julio 2009 - Abril 2010 se halló que los servicios que mayormente derivan a la Residencia son los de: Reumatología y Clínica Médica (Portela, Illán, Hernández, Muñoz, Caillet-Bois 2010); se buscó conocer la demanda institucional pero por motivos de practicidad no se tomó en cuenta la respuesta brindada por la misma. A través del presente trabajo buscamos conocer el proceso de Terapia Ocupacional ${ }^{6}$ (evaluación, intervención y reevaluación de resultados para posible alta de tratamiento) (AOTA, 2002) en Internación de Clínica Médica y Unidad de Terapia Intermedia y Consultorios Externos de Reumatología.

No se pretende extrapolar los resultados obtenidos a la población general sino describir la atención brindada a los pacientes atendidos por la Residencia de Terapia Ocupacional del Hospital Gral. de Agudos Dr. E. Tornú.

Objetivo general:

Conocer el proceso de tratamiento de Terapia Ocupacional en las áreas de consultorios externos de Reumatología e internación de Clínica Médica y Terapia Intermedia del Hospital de Agudos Dr. E. Tornú en el periodo de Junio a Diciembre 2010.

Objetivos específicos:

- Determinar la prevalencia de los diferentes motivos de internación/ diagnósticos prevalentes en ambas áreas.

- Determinar la prevalencia de cada intervención en ambas áreas.

- Medir la cantidad de sesiones brindadas por paciente en ambas áreas.

- Determinar la cantidad de intervenciones por paciente en ambas áreas.

- Determinar la prevalencia de los motivos de alta en los pacientes en ambas áreas.

- Comparar la prevalencia de intervenciones por paciente según edad en ambas áreas.

\footnotetext{
${ }^{6}$ Según el "Marco de Trabajo para la práctica de T.O.: Ámbito de competencia y Proceso" (Marco de Trabajo), el Proceso de tratamiento de T.O. empieza por la evaluación de las necesidades ocupacionales, problemas y prioridades del cliente. Proporciona un conocimiento de la historia ocupacional.
}

El plan de intervención son las acciones que se toman para influir y dar soporte en la mejora del desempeño del cliente. Para finalizar la reevaluación de los resultados se usa como información para planificar acciones futuras con el cliente y evaluar el programa de servicio. 


\section{METODO}

Diseño del protocolo: se realizó un estudio descriptivo y comparativo, observacional, retrospectivo, transversal, cuantitativo.

Población y muestra:

Unidad de análisis: todos los pacientes asistidos por la Terapia Ocupacional en las áreas de internación de Clínica Médica., Unidad de Terapia Intermedia y en consultorios externos de Reumatología durante el período Julio - Diciembre 2010.

Variables:

TIPOS DE INTERVENCIONES: se tomaron las intervenciones con su definición teórica acorde al Marco de Trabajo $^{7}$ ( $2^{\circ}$ edición) (AOTA, 2008) complementando con el Instructivo de la ficha interna de evolución de la Residencia de Terapia Ocupacional: "uso terapéutico de uno mismo", "uso terapéutico de las ocupaciones y actividades", "asesoría" y "formación", aplicándolas a nuestro entorno hospitalario ${ }^{8}$. En cada paciente se registró si presentó o no dichas intervenciones.

MOTIVOS DE ALTA: causa que genera la finalización de la relación terapéutica entre paciente y Terapista Ocupacional, las cuales son: Óbito, Abandono de tratamiento, Negatividad de la persona a recibir o continuar tratamiento, Cumplimiento de objetivos de tratamiento de Terapia Ocupacional, Alta médica de internación, Pase de sala donde no hay asistencia de la Residencia.

CANTIDAD DE SESIONES: por pacientes.

EDAD: edad en años cumplidos, tomada de la fecha de nacimiento que figura en la Historia Clínica $(\mathrm{HC})$.

\footnotetext{
${ }^{7}$ En la 2o edición del Marco de Trabajo se mencionan las mismas intervenciones que en su antecesor, incorporando únicamente la Auto-gestión, la cual no la tomaremos debido a que dicha intervención no se realiza en la RTO.

${ }^{8}$ Ficha de evolución interna de la Residencia de Terapia Ocupacional, versión 2010.
} 
DIAGNÓSTICO: se categorizaron según tipo de patología en Internación, de acuerdo al sistema corporal afectado.

Fuente e instrumento:

Primaria: recopilación documental de las Fichas de registro de evaluación y de evolución internas de la Residencia de Terapia Ocupacional ${ }^{9}{ }^{10}$. Para obtener los datos se registraron todas las intervenciones realizadas en todos los pacientes atendidos durante el periodo anteriormente mencionado.

PLAN DE ANÁLISIS: los datos se registraron en una planilla de cálculo Excel y se analizaron la cantidad y prevalencia de cada una de las variables de acuerdo a los objetivos del trabajo. Por otro lado se realizó una estadística analítica donde se utilizó el método chicuadrado para realizar las comparaciones mediante el software SPSS versión 17. Para evitar sesgo en el registro de datos, se realizó una doble entrada de los datos en la planilla de excel.

REPAROS ÉTICOS: este estudio forma parte del Programa de formación de la Residencia de Terapia Ocupacional; el mismo se llevó a cabo sin recibir compensación económica alguna y sin fines de lucro. Fue aprobado por el Comité de Ética en Investigación y por el Comité de Docencia e Investigación del Hospital. Los investigadores se comprometen moral y éticamente a respetar la confidencialidad en el manejo de los datos recopilados, en base a la norma Helsinski y a la ley de "Protección de los datos personales" Nro. 25326.

\section{RESULTADOS:}

Se atendieron 140 pacientes en el periodo Junio-Diciembre 2010 y se realizaron un total de 1.755 intervenciones en ambas áreas con el $67 \%$ correspondiente a Internación. La Mediana fue de 11 intervenciones por paciente y la Media de 18 intervenciones (rango 1-80) en Internación.

En Reumatología la Mediana fue de 6 intervenciones por paciente y la Media de 8 intervenciones (rango 1-45) .

\footnotetext{
${ }^{9}$ Ficha de evaluación interna de Clínica Médica y Unidad de Terapia Intermedia de la Residencia de Terapia Ocupacional, versión 2010.

${ }^{10}$ Ficha de evaluación interna de Reumatología de la Residencia de Terapia Ocupacional, versión 2010.
} 
La intervención utilizada con más frecuencia fue el Uso terapéutico de las actividades y ocupaciones con un 41 \% en Internación y 47 \% en Reumatología. Es notable la diferencia en la cantidad de IC realizadas con un $28 \%$ en Internación (más del $50 \%$ de las mismas realizadas para solicitar información) y un $5 \%$ en Reumatología. El Uso terapéutico del Yo fue la intervención menos frecuente en ambas áreas (5\%) (Gráfico № 1)

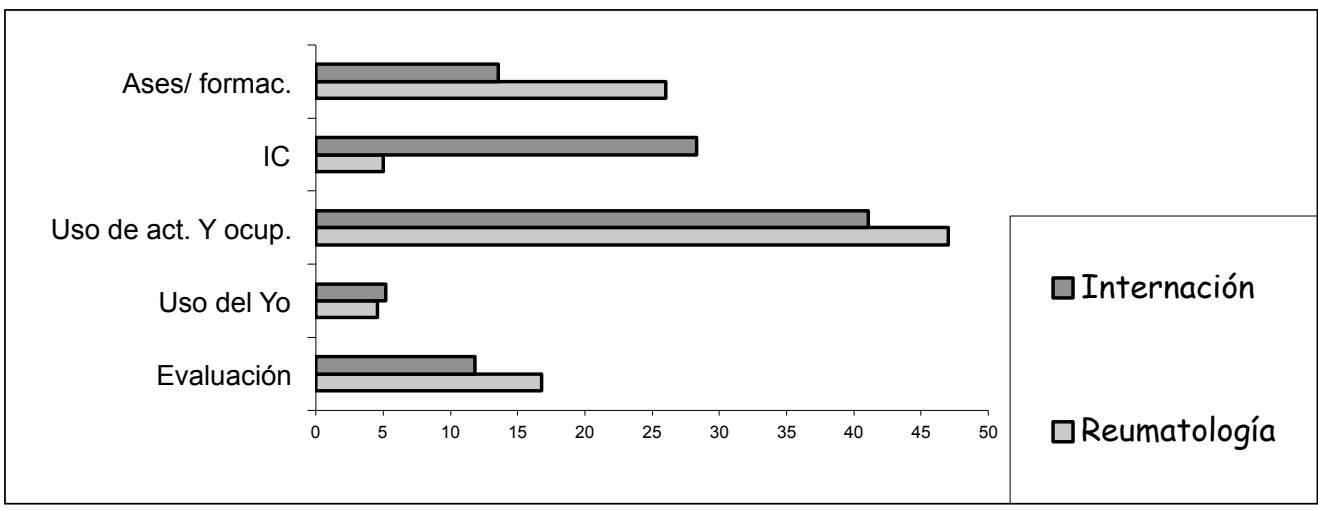

Gráfico № 1: Prevalencia de intervenciones en internación y reumatología.

En Reumatología se observa una diferencia estadísticamente significativa en la intervención: Utilización terapéutica de actividades y ocupaciones (uso de actividades propositivas) según la edad con mayor prevalencia en el grupo de 54 a 66 años y menor en el grupo de 44 a 53 años $(p=0,023)$. No observamos diferencias significativas $(p>0,05)$ entre grupos de otras edades y las restantes intervenciones así como tampoco en el área de Internación

Se realizaron 880 sesiones en total de las cuales el 71\% corresponde a Internación. La Mediana fue de 6 sesiones por paciente y la Media de 9 sesiones (rango 1-47) y solo el 25\% recibió más de 15 sesiones en internación. En Reumatología la Mediana fue de 3 sesiones por paciente y la Media de 3,5 (rango 1-14) sesiones.

En la intervención Uso terapéutico de las actividad y ocupaciones, se destacan los métodos preparatorios en ambas áreas con un 56\% en Internación y un 84 \% en Reumatología. De los mismos en Internación se destacan los ejercicios (tanto físicos como cognitivos) y el posicionamiento; y en Reumatología se destaca la confección de férulas y la realización de ejercicios físicos. (Gráfico $N^{\circ} 2$ ) 
Gráfico $N^{\circ}$ 2: Prevalencia de Uso terapéutico de las actividad y ocupaciones en ambas áreas.

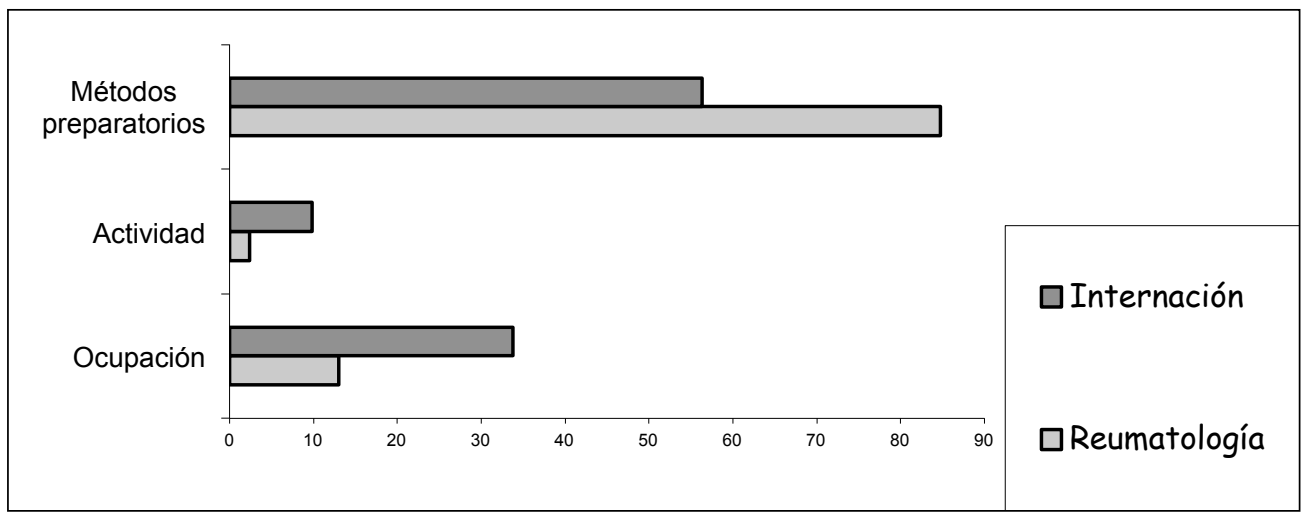

En relación a las patologías en Internación la de mayor prevalencia fue Neurológicas con el $42 \%$, seguida de Musculoesqueléticas y Cardiológicas con el $15 \%$ y $12 \%$ respectivamente; en Reumatología fue Osteoartrosis con el 27,4\% seguida de Artritis Reumatoidea con el $24,6 \%$ y Síndrome del Túnel Carpiano con el 16,4\%.

El motivo de alta más frecuente en Internación fue alta médica con el $56 \%$ (n:43), seguido de cumplimiento de objetivos con el $16 \%$ ( $n: 10$ ), y el menos frecuente fue Negativa: el $1 \%$ (n: 1).

En Reumatología solo se registraron dos motivos abandono de tratamiento con el $65 \%$ ( $\mathrm{n}$ : 43) y cumplimiento de objetivos con el $34 \%$ (n: 22). (Gráfico $N^{\circ} 3$ )

Al momento del análisis de los datos en ambas áreas se encontraban paciente que estaban en tratamiento (8 pacientes en Reumatología y 7 en Internación).

\section{Gráfico № 3: Motivos de alta en Internación y Reumatología}

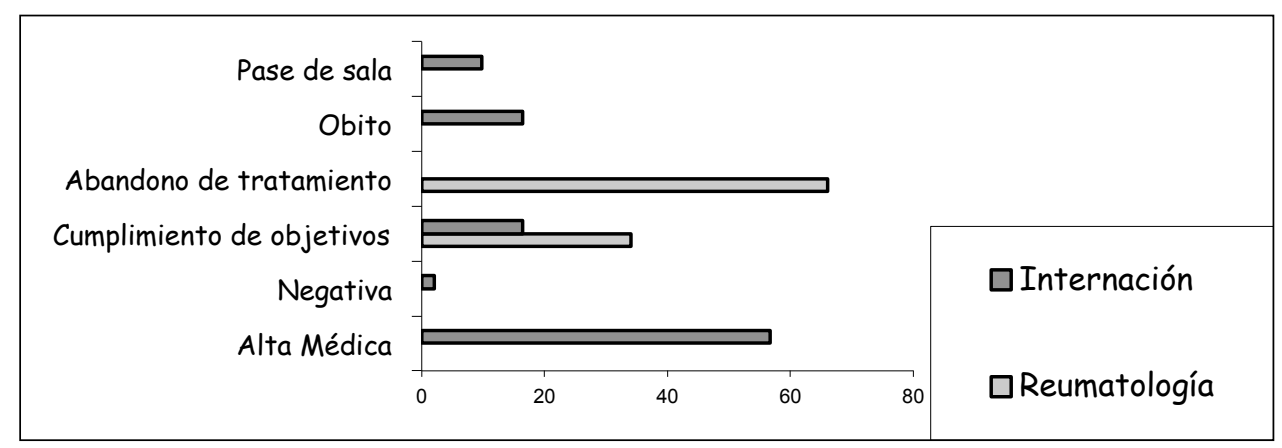




\section{DISCUSIÓN}

\section{Limitaciones del trabajo:}

Para determinar la prevalencia de patologías en Internación se tomó en cuenta el diagnóstico por el cual cada paciente fue derivado a Terapia Ocupacional, sin embargo la población atendida es adulta y presenta múltiples comorbilidades que asociado al desacondicionamiento producido por la hospitalización prolongada, afecta en mayor o menor medida, la capacidad funcional del paciente al momento de nuestra evaluación inicial. Las mismas no se tuvieron en cuenta al momento de la clasificación. Se sugieren para próximos estudios tener en cuenta el diagnóstico del paciente escrito en la Historia Clínica, el diagnóstico y motivo de derivación a Terapia Ocupacional, y las comorbilidades presentes. Al tener en cuenta estos factores se podrán correlacionar los mismos con nuestras intervenciones realizadas.

El tipo de estudio realizado es retrospectivo, por lo cual los datos analizados fueron registrados previo al diseño del trabajo, con la posibilidad de crear sesgos en el análisis de los resultados y de este modo afectar la validez y confiabilidad del trabajo. En esta misma línea, se sugiere replicar este estudio de manera prospectiva a fin de mejorar la toma de datos y de esta manera la calidad metodológica del trabajo.

El instrumento de evaluación "Ficha de evolución" utilizado fue confeccionado en mayo del 2010 y se implementó en junio del corriente año, al mismo tiempo en que se inició la toma de datos. Lo mismo ocurrió con la categorización de intervenciones, dado que previamente no se utilizaba ninguna clasificación determinada. Ambos son métodos de trabajo nuevos, no existió un proceso de aprendizaje previo a este estudio y por este motivo creemos que pueden existir sesgos en la toma de datos, creando un subregistro de nuestras intervenciones como ser cantidad de Interconsultas que realizamos, el Uso terapéutico del Yo que prima cuando se interviene en una situación de gran estrés como es una enfermedad y su internación, y la re-evaluación que realizamos cada vez que comenzamos una nueva sesión de tratamiento.

\section{Algunas consideraciones:}

De acuerdo a la bibliografía consultada (Pérez 2006; Illán, Tommasi 2010) son numerosas las intervenciones desde Terapia Ocupacional en internación que tienen por objetivo evitar complicaciones secundarias a la hospitalización; restaurar y mantener el funcionamiento; optimizar la recuperación y autonomía precoz: actividades de la vida diaria, adaptaciones y 
equipamiento, educar al paciente y/o familiar, realizar Interconsultas para vehiculizar necesidades del paciente, etc.

En nuestro trabajo, el uso terapéutico de la actividad y ocupaciones, fue la intervención más prevalente en ambas áreas. Dicha intervención es propia y característica de la disciplina, siendo el fin último de la misma, el desempeño óptimo de cada persona según sus prioridades y en relación a su capacidad funcional. Es importante aclarar que la prevalencia de esta intervención en ambas áreas se debe al gran porcentaje de métodos preparatorios realizados, entendidos éstos como intervenciones necesarias para preparar y facilitar a la persona para la funcionalidad en sus ocupaciones, ya sea que se utilicen en forma temporal o permanente; dicha intervención es aún mayor en Reumatología dado que se vincula directamente con el alivio del síntoma que se encuentra limitando al desempeño en las ocupaciones.

No se consideró la Interconsulta para pedir y/o transmitir información mediante la lectura y escritura de las Historia Clínica como un tipo de intervención a la hora de registrarlo en la evolución. La misma es la principal vía de comunicación con el equipo de trabajo, principalmente en consultorios externos de Reumatología. En la sala de internación la comunicación verbal e informal se da con mayor frecuencia dado que es el lugar de encuentro de las diferentes disciplinas junto con el paciente, es decir ambos entornos, físico y temporal, actúan como facilitadores para las Interconsultas. Sin embargo, a partir del análisis de los datos se observa que dicha intervención no se ha realizado con la frecuencia necesaria que requiere la complejidad del paciente agudo, por lo que consideramos relevante plantear nuevas estrategias de comunicación con el equipo de salud, en particular en consultorios externos, a fin de propiciar una comunicación fluida y mejorar la calidad de nuestras intervenciones.

Según los resultados obtenidos creemos que existen diversos factores que influyen en que una intervención pueda ser realizada con mayor o menor frecuencia, por ejemplo la continua rotación de residentes en formación por las áreas de la Residencia sin un profesional de planta permanente; la subjetividad que cada Terapista Ocupacional tiene al momento de evolucionar, priorizando una intervención en detrimento de otra; la posibilidad de realizar ocupaciones en el área de Reumatología respecto a Internación, debido a las características del entorno físico dado que el consultorio externo puede ser menos propicio para la ejecución de las ocupaciones; la variabilidad del cuadro clínico en el paciente agudo que obliga a que se deba suspender determinada intervención o bien repetirse por mayor tiempo; la existencia de red social continente que acompañe y aplique nuestras intervenciones y sugerencias, etc. 
En Internación se intenta que la frecuencia de tratamiento sea diaria. Consecuentemente es también mayor la cantidad de intervenciones y sesiones registradas en comparación con Reumatología donde la frecuencia de tratamiento es principalmente semanal. Esto también se encuentra en relación a los motivos de alta, ya que es difícil o muy poco frecuente que durante un momento agudo de enfermedad el paciente internado rechace el tratamiento de Terapia Ocupacional a diferencia de Reumatología donde el abandono de tratamiento es la causa prevalente del alta.

En el trabajo de investigación "Perfil de Interconsultas a Terapia Ocupacional" se establecen las características del paciente tipo derivado de cada servicio; en Internación se describe a una mujer de 74 años, jubilada, con una patología neurológica derivada para movilización y en Reumatología a una mujer de 55 años, empleada doméstica, con diagnóstico de Artritis Reumatoidea, derivada para confección de férulas. Como se puede inferir de lo anteriormente dicho, la persona que concurre al consultorios externos está laboralmente activa, con una patología crónica, cuyo principal interés es el alivio del síntoma para poder continuar con su rutina, por lo cual una vez logrado el objetivo abandona el tratamiento (a pesar de contar con otros objetivos no agudos para seguir trabajando) y retorna sólo en caso de reagudización del síntoma molesto. Esto se diferencia del paciente internado donde el motivo de alta más frecuente es alta médica. Creemos necesario reformular la modalidad de atención por consultorios externos a fin de lograr mayor adherencia del paciente al tratamiento. En relación a la Internación, sería óptimo poder planificar al alta conjuntamente con el médico tratante de modo de prevenir reingresos a corto plazo.

\section{CONCLUSION}

Consideramos que se ha logrado cumplir con los objetivos del trabajo pretendiendo dar cuenta de la modalidad de intervención de Terapia Ocupacional en este hospital de agudos, considerando la misma como un proceso de evaluación, intervención y resultados.

Dado el subregistro de intervenciones observado en las fichas de evolución interna, es necesario mejorar la metodología en la utilización de la misma a fin de reflejar la real frecuencia con que se realizan las intervenciones.

La importancia de los datos obtenidos radica en la posibilidad de contribuir a mejorar la atención de los pacientes asistidos en el Hospital E. Tornú por la Residencia de Terapia Ocupacional. Sin embargo, se requieren de estudios más exhaustivos que profundicen los 
datos obtenidos, teniendo en cuenta no sólo las intervenciones realizadas sino la percepción o satisfacción del paciente con las mismas.

\section{AGRADECIMIENTO}

Se agradece a la Dr. Diana S. Klajn por su colaboración para el desarrollo del presente trabajo.

\section{REFERENCIAS BIBLIOGRÁFICAS}

American Occupational Therapy Association (2002). Occupational Therapy Practice Framework: Domain and process. AJOT. LIV. № 6. 609-639. Traducción y adaptación al español realizada por: Mantilla Morta R. y cols. (diciembre 2005).

American Occupational Therapy Association (2008). Occupational Therapy Practice Framework: Domain and process, AJOT. LXII. 625-683. Traducción y adaptación al español realizada por: Mercado Padín R., Ramírez Ríos A. y cols. (abril 2010).

Illán V., Tommasi L. (2010). Características de los pacientes internados en el Hospital General de Agudos Dr,. E. Tornú y necesidades de intervención de Terapia Ocupacional. Revista científica Aesculapius. № 1. 19-26.

Pérez M. (2006). Terapia Ocupacional en un Hospital General de Agudos. Observaciones no publicadas. Buenos Aires, Argentina.

Portela A., Illán V., Hernández E., Muñoz P., Caillet-Bois C. (2010) Perfil de Interconsulta a Terapia Ocupacional. Recuperado en marzo 2011 en http://www.terapiaocupacional.com.ar 